\title{
ON CANONICAL CONFORMAL MAPS OF MULTIPLY CONNECTED DOMAINS
}

\author{
BY \\ H. J. LANDAU
}

In a recent paper [11], J. L. Walsh has established the existence of two new canonical domains for the conformal mapping of multiply connected regions. The canonical maps may be characterized by the fact that they transform certain harmonic domain functions into functions which are extendible harmonically to the entire plane, with the exception of a finite number of points, one in each of the components of the complement of the canonical domain. Two other existence proofs have been given, by H. Grunsky [6] and J. A. Jenkins [7]. All of these proofs rely on uniformization. In part I of the present paper we give a geometric and constructive proof of existence, based primarily on potential-theoretic considerations. In part II we consider the relation between the new canonical mapping functions and extremal problems, and show thereby that the maps are natural generalizations of those which take, respectively, a doubly connected domain onto an annulus and simply connected domain onto the unit circle. Finally, in part III, by specializing the existence proof to the case of a doubly connected domain, we obtain a new construction procedure for the conformal map of such a domain onto an annulus, and show it to converge more rapidly than one commonly used at present.

The author is deeply grateful to Professors J. L. Walsh, L. V. Ahlfors, and R. Osserman for their inspiration and help.

I. Existence of the canonical map. Throughout this discussion let the term "harmonic measure of an annular domain" be taken to mean the harmonic measure of the outer boundary curve with respect to the domain; that is, the function which is harmonic in the domain and takes the values 0,1 on the inner and outer boundary curves respectively. Also, let the term "contour" denote an analytic Jordan curve.

Theorem 1. Let $C_{1}$ be a Jordan curve in the z-plane, and $D$ its exterior. Let $C$ be an arbitrary contour in $D$ containing $C_{1}$ in its interior, and let $D^{\prime} \subset D$ denote the doubly connected region bounded by $C_{1}$ and $C$. There exists a map $w=f(z)$, one to one and conformal in $D$ and mapping $D$ onto the exterior of $a$ contour $\gamma_{1}$, such that the harmonic measure of the image of $D^{\prime}$ is extendible harmonically to the interior of $\gamma_{1}$ except for a single point, where it has a logarithmic singularity. This map is unique up to a linear transformation.

Presented to the Society, January 30, 1958; received by the editors December 2, 1959. 
Proof. We will construct a sequence of maps which extend the harmonic measure farther and farther into the interior of the image of $C_{1}$. We will then show that the limit of this sequence satisfies the requirements of Theorem 1.

A. Construction of the sequence. We may assume that $C_{1}$ is the unit circumference, for $C_{1}$ may always be mapped onto $|z|=1$ by a preliminary transformation one to one and conformal exterior to $C_{1}$, and taking $\infty$ onto $\infty$. Let $R=\min _{z \in c}|z|$; we have $R>1$. The harmonic measure $u(z)$ of $D^{\prime}$ is extendible by reflection up to $C_{r}$, the reflection of $C$ in $|z|=1$, which lies interior to $|z|=1 / R$.

Let first $w_{1}^{*}=g_{1}^{*}(z)$ be the conformal map of the exterior of $C_{r}$ onto $\left|w_{1}^{*}\right|>1 / R$, with $g_{1}^{*}(\infty)=\infty$. Let $C^{*}$ be the image of $C$ under $g_{1}^{*}(z)$, and let $g_{1}^{*}(z)=a_{0}+a_{1} z+b_{1} / z+b_{2} / z^{2}+\ldots$ in a neighborhood of $z=\infty$. By Schwarz's Lemma we have $\left|a_{1}\right| \geqq 1$ and $\left|w_{1}^{*}\right| \geqq|z|$ for all $z$ exterior to $C_{r}$; thus, setting $R_{1}=\min _{w_{1}} \in C^{*}\left|w_{1}^{*}\right|$, we obtain $R_{1} \geqq R$.

It is important, however, to normalize the map at $\infty$. We set $w_{1}=g_{1}(z)$ $\equiv g_{1}^{*}(z) / a_{1}$, let $C^{(1)}$ be the image of $C$ under $w_{1}=g_{1}(z)$, and $K_{1}=1 /\left|a_{1}\right| \leqq 1$. The net result is

$$
w_{1}=\sigma_{0}^{(1)}+z+\frac{\sigma_{1}^{(1)}}{z}+\frac{\sigma_{2}^{(1)}}{z^{2}}+\cdots,
$$

The map $w_{1}=g_{1}(z)$ is one to one and conformal exterior to $C_{r}$, and takes the exterior of $C_{r}$ onto $\left|w_{1}\right|>K_{1} / R$ and $C$ onto a contour $C^{(1)}$ which contains in its interior the disk $\left|w_{1}\right|<K_{1} R_{1}$. The function $w_{1}(z)-z$ is analytic without singularities exterior to $C_{r}$, including at $z=\infty$, and thus by the maximum principle

$$
\begin{aligned}
\left|w_{1}-z\right| & \leqq\left[\left|w_{1}(z)-z\right|, \text { for } z \text { in } C_{r} \mid\right. \\
& \leqq\left|w_{1}\right|+|z|, \text { for } z \text { in } C_{r} \\
& \leqq K_{1} / R+1 / R=\left(K_{1}+1\right) / R .
\end{aligned}
$$

Now again, by reflection, the harmonic measure is extendible up to $C_{r}^{(1)}$, the reflection of $C^{(1)}$ in $\left|w_{1}\right|=K_{1} / R$, which lies interior to $\left|w_{1}\right|=K_{1} / R^{2} R_{1}$.

Iterating this procedure, we may define $(n=2,3,4, \cdots)$

$$
w_{n}=g_{n}\left(w_{n-1}\right)=\sigma_{0}^{(n)}+w_{n-1}+\frac{\sigma_{1}^{(n)}}{w_{n-1}}+\frac{\sigma_{2}^{(n)}}{w_{n-1}^{2}}+\cdots
$$

in a neighborhood of $w_{n-1}=\infty$.

The map $w_{n}=g_{n}\left(w_{n-1}\right)$ is one to one and conformal exterior to $C_{r}^{(n-1)}$, and it takes the exterior of $C_{r}^{(n-1)}$ onto $\left|w_{n}\right|>K_{1} K_{2} \cdots K_{n} / R_{n-1} R_{n-2}^{2} \cdots$ $R_{1}^{2^{(n-2)}} R^{2^{(n-1)}}$, with $R_{n-1}>R_{n-2} \cdots>R_{1}>R>1$ and $K_{i} \leqq 1$; the image under this map of $C^{(n-1)}$ is a contour $C^{(n)}$ which contains in its interior the disk 
$\left|w_{n}\right|<K_{1} K_{2} \cdots K_{n} R_{n}$ where $R_{n} \geqq R_{n-1}$. The function $w_{n}\left(w_{n-1}\right)-w_{n-1}$ is analytic without singularities exterior to $C_{r}^{(n-1)}$, including at $w_{n-1}=\infty$, and thus by the maximum principle

$$
\begin{aligned}
\left|w_{n}-w_{n-1}\right| & \leqq\left[\left|w_{n}\left(w_{n-1}\right)-w_{n-1}\right|, \text { for } w_{n-1} \text { in } C_{r}^{(n-1)}\right] \\
& \leqq\left|w_{n}\right|+\left|w_{n-1}\right|, \text { for } w_{n-1} \text { in } C_{r}^{(n-1)} \\
& \leqq K_{1} K_{2} \cdots K_{n-1}\left(K_{n}+1\right) / R_{n-1} R_{n-2}^{2} \cdots R^{2^{(n-1)}} .
\end{aligned}
$$

By reflection, the harmonic measure will be extendible up to $C_{r}^{(n)}$, the reflection of $C^{(n)}$ in $\left|w_{n}\right|=K_{1} \cdots K_{n} / R_{n-1} R_{n-2}^{2} \cdots R^{2^{(n-1)}}$, which lies interior to $\left|w_{n}\right|=K_{1} \cdots K_{n} / R_{n} R_{n-1}^{2} \cdots R_{1}^{2^{(n-1)}} R^{2^{n}}$.

B. Limit OF THE SEQUenCE. Consider now the mappings $w_{n}=g_{n}\left(w_{n-1}\right)$ $=f_{n}(z)$ all as functions of $z$ defined, one to one, and conformal in the domain $E$, consisting of the exterior of the contour $C_{r}$. By the normalization, $w_{n}=f_{n}(z)$ $=A_{0}^{(n)} / z+A_{1}^{(n)} / z+A_{2}^{(n)} / z^{2}+\cdots$ in a neighborhood of $z=\infty$. Thus all the functions $f_{n}(z)-z$ are analytic without singularities in the entire domain $E$ and we have by (1)

$$
\begin{aligned}
& \left|f_{n}(z)-z\right|=\left|w_{n}-w_{n-1}+w_{n-1}-w_{n-2}+\cdots+w_{1}-z\right| \\
& \leqq\left|w_{n}-w_{n-1}\right|+\left|w_{n-1}-w_{n-2}\right|+\cdots+\left|w_{1}-z\right| \\
& \leqq \frac{K_{1} K_{2} \cdots K_{n-1}\left(K_{n}+1\right)}{R_{n-1} R_{n-2}^{2} \cdots R_{1}^{2^{(n-2)}} R^{2^{(n-1)}}}+\cdots+\frac{K_{1}\left(K_{2}+1\right)}{R_{1} R^{2}}+\frac{K_{1}+1}{R} \\
& \leqq B
\end{aligned}
$$

Hence the functions $f_{n}(z)-z$ form a normal family in $E$.

Consider the infinite sequence of functions:

$$
f_{1}(z)=\left[f_{1}(z)-z\right]+z ; \quad f_{2}(z)=\left[f_{2}(z)-z\right]+z ; \quad \ldots
$$

all one to one and conformal in $E$. Select first a subsequence $f_{m_{1}}(z), f_{m_{2}}(z), \ldots$ such that the sequence of functions: $\left[f_{m_{1}}(z)-z\right],\left[f_{m_{2}}(z)-z\right], \cdots$ converges uniformly on every compact (on the Riemann sphere) subset of $E$. Then $f(z)=\lim _{i \rightarrow \infty}\left[f_{m_{i}}(z)-z\right]+z$ is a nonconstant, hence univalent, analytic function on $E$.

We will have occasion, in the subsequent discussion, to use the following elementary lemma, whose statement and proof we reproduce from [11].

LEMMA 1. Let $g_{n}(z)$ be a sequence of functions analytic and univalent in a domain $R$, converging uniformly on every compact subset of $R$ to a function $g(z)$ which is nonconstant and therefore univalent. Let $S$ be the image of $R$ under the map $w=g(z)$, and $S^{*}$ be a compact subset of $S$. Then, for all $n$ sufficiently large, the image of $R$ under the map $w=g_{n}(z)$ covers $S^{*}$.

Proof of Lemma 1. Let $\Gamma$ be a Jordan curve, or the union of a finite num- 
ber of Jordan curves in $R$, whose image under the map $w=g(z)$ lies in $S-S^{*}$ and separates $S^{*}$ from the boundary of $S$. Since $\Gamma$ forms a compact subset of $R$, we have $g_{n}(z) \rightarrow g(z)$ uniformly on $\Gamma$, thus for $z$ in $\Gamma$ and all $n$ sufficiently large we have, uniformly for $w$ in $S^{*}$,

$$
\left|\frac{g_{n}(z)-g(z)}{g(z)-w}\right|<1,
$$

whence by Rouché's theorem, $g_{n}(z)-w$ has the same number of zeros in the region bounded by $\Gamma$ as does $g(z)-w$, namely one. Lemma 1 is established.

Denote now by $f_{n}(z)$ the subsequence selected above which converges to $f(z)$ on $E$, uniformly on every compact (on the Riemann sphere) subset of $E$. Let the map $w=f(z)$ take the domains $D$ and $D^{\prime}$ onto domains $\Delta$ and $\Delta^{\prime}$ respectively, and the contours $C_{1}:|z|=1$ and $C$ onto contours $\gamma_{1}$ and $\gamma$ respectively. Similarly, let the map $w=f_{n}(z)$ take the domains $D$ and $D^{\prime}$ onto domains $\Delta_{n}$ and $\Delta_{n}^{\prime}$ respectively. By Lemma 1 , the curve $\gamma$ is contained in the domain $\Delta_{n}$ for some $n$ sufficiently large and so, by construction of the $\Delta_{n}$, does not pass through the point $w=0$. Furthermore, since on the compact subset $C$ of $|z|>1$ the functions $f$. (z) converge uniformly to $f(z)$ we have

$$
[\arg f(z)]_{C}=\int_{C} \frac{g^{\prime}(z)}{g(z)} d z=\lim _{n \rightarrow \infty} \int_{C} \frac{g_{n}^{\prime}(z)}{g_{n}(z)} d z=\lim _{n \rightarrow \infty}\left[\arg f_{n}(z)\right]_{C},
$$

and $\left[\arg f_{n}(z)\right]_{C}=2 \pi$ for all $n$, by construction of the maps $w=f_{n}(z)$. It follows that the contour $\gamma$ separates $w=0$ from $w=\infty$.

Select next a region $D_{0}$ whose closure $\bar{D}_{0}$ is a compact subset of $D^{\prime}$, and let $\Delta_{0}$ be the image of $D_{0}$ under the map $w=f(z)$. By Lemma 1 , any compact subset of $\Delta_{0}$ is contained in all the domains $f_{n}\left(D_{0}\right)$ for $n$ sufficiently large. Thus the maps $z=f_{n}^{-1}(w)$ are defined and univalent on every compact subset of $\Delta_{0}$, for $n$ sufficiently large, and map it into $D_{0}$; they therefore form a normal family in $\Delta_{0}$, and we may restrict ourselves to a subsequence which approaches a limit $F(w)$ on $\Delta_{0}$, uniformly on every compact subset of $\Delta_{0}$. The function $F(w)$ is either univalent or identically constant on $\Delta_{0}$. On any given compact subset of $\Delta_{0}$, the function $F(w)$ is the uniform limit of the functions $f_{n}^{-1}(w)$ so that the image of that compact subset under the map $z=F(w)$ is the limit of its images under the maps $z=f_{n}^{-1}(w)$. But the maps $z=f_{n}^{-1}(w)$, as soon as $n$ is sufficiently large, map the given subset into $D_{0}$, hence their limit must be contained in $\bar{D}_{0}$. We conclude that the image of $\Delta_{0}$ under the map $z=F(w)$ is contained in $\bar{D}_{0}$. Thus, for $w_{0} \in \Delta_{0}$, we have $\lim _{n \rightarrow \infty} f_{n}^{-1}\left(w_{0}\right)=F\left(w_{0}\right) \in \bar{D}_{0}$ so that for $n$ sufficiently large all the points $f_{n}^{-1}\left(w_{0}\right)$ lie in a compact subset of $D^{\prime}$, where the functions $f_{n}(z)$ converge uniformly to $f(z)$. Then by Carathéodory's criterion for continuous convergence [4], we have $f\left[F\left(w_{0}\right)\right]$ $=\lim _{n \rightarrow \infty} f_{n}\left[f_{n}^{-1}\left(w_{0}\right)\right]=w_{0}$. Similarly, for $z_{0} \in D_{0}$, we have $\lim _{n \rightarrow \infty} f_{n}\left(z_{0}\right)=f\left(z_{0}\right)$ $\in \Delta_{0}$, so that for $n$ sufficiently large all the points $f_{n}\left(z_{0}\right)$ lie in a compact subset of $\Delta_{0}$, where the functions $f_{n}^{-1}(w)$ converge uniformly to $F(w)$. Therefore, 
as above, $F\left[f\left(z_{0}\right)\right]=\lim _{n \rightarrow \infty} f_{n}^{-1}\left[f_{n}\left(z_{0}\right)\right]=z_{0}$. Consequently $F(w)$ is not constant and coincides with the inverse function $f^{-1}(w)$ of $f(z)$, for $w \in \Delta_{0}$ and $z \in D_{0}$.

Let $u(z)$ be, as before, the harmonic measure of $D^{\prime}$ (i.e., the harmonic measure of the contour $C$ with respect to $\left.D^{\prime}\right)$; similarly, let $v(w)$ and $v_{n}(w)$ be the harmonic measures respectively of the domains $\Delta^{\prime}$ and $\Delta_{n}^{\prime}$. On $\Delta_{n}^{\prime}$ we have $v_{n}(w) \equiv u\left[f_{n}^{-1}(w)\right]$, since both functions are harmonic in $\Delta_{n}^{\prime}$ and assume the same values on the boundary of $\Delta_{n}^{\prime}$; similarly, $v(w) \equiv u\left[f^{-1}(w)\right]$.

Denote by $R$ the domain bounded by the contour $\gamma$ and the point $w=0$. By Lemma 1 , every compact subset of $\Delta^{\prime}$ lies in all the $\Delta_{n}^{\prime}$, for $n$ sufficiently large; furthermore, by construction, the function $v_{n}(w)$ is extendible har. monically across the inner boundary of $\Delta_{n}^{\prime}$, up to the curve $C_{r}^{(n)}$, which lies interior to $|w|=K_{1} \cdots K_{n} / R_{n} \cdots R^{2^{n}}$. Hence the functions $v_{n}(w)$ are defined and harmonic on any given compact subset of $R$ for $n$ sufficiently large, and $v_{n}(w)<1$ everywhere in $R$. The functions $v_{n}(w)$ thus form a normal family in $R$, and we may find a subsequence converging on $R$, uniformly on every compact subset of $R$, to a limiting function $V(w)$ which is either harmonic in $R$ or identically equal to $-\infty$. The latter cannot be the case, since from the preceding discussion, on the subdomain $\Delta_{0}$ of $R, \lim _{n \rightarrow \infty} f_{n}^{-1}(w)$ $=f^{-1}(w)$, so that, since $u(z)$ is continuous, we have for $w \in \Delta_{0}$

$$
V(w)=\lim _{n \rightarrow \infty} v_{n}(w) \equiv \lim _{n \rightarrow \infty} u\left[f_{n}^{-1}(w)\right]=u\left[f^{-1}(w)\right] \equiv v(w) \neq-\infty .
$$

Thus $V(w)$ is harmonic in $R$. But since, as we have just shown, the harmonic functions $V(w)$ and $v(w)$ coincide on the open subdomain $\Delta_{0}$ of $\Delta^{\prime}$, they coincide everywhere on their common domain of definition $\Delta^{\prime}$, and the function $V(w)$ is seen to be the required harmonic extension of the harmonic measure $v(w)$ of $\Delta^{\prime}$ (the image of $D^{\prime}$ under the map $w=f(z)$ ) to the interior of $\gamma_{1}$, with the exception of the single point $w=0$. Since the harmonic function $V(w)$ approaches $-\infty$ in the neighborhood of $w=0$, it is of the form $A \log |w|$ $+V_{1}(w)$, with $V_{1}(w)$ harmonic in the neighborhood of $w=0[10$, p. 675]. We have $A=\int_{\gamma_{1}} \partial V / \partial n d s=\int_{\gamma} \partial v / \partial n d s ; A$ is therefore a conformal invariant, equal to the period of the conjugate function of the harmonic measure of $D^{\prime}$ around any curve in $D^{\prime}$ homologous to $C$.

C. Uniqueness of THE MAP. Suppose that $w=f(z)$ and $W=F(z)$ are two conformal maps of $D$ onto the exteriors of contours $\gamma_{1}$ and $\Gamma_{1}$ in the $w$ and $W$. planes respectively, such that the harmonic measures $v(w)$ and $V(W)$ of the images $\delta^{\prime}$ and $\Delta^{\prime}$ of $D^{\prime}$ are extendible harmonically everywhere to the interiors of $\gamma_{1}$ and $\Gamma_{1}$, with the exception of the points $w_{0}$ and $W_{0}$, at which they have $\operatorname{logarithmic}$ singularities $A \log \left|w-w_{0}\right|$ and $A \log \left|W-W_{0}\right|$; the coefficient $A$ is necessarily the same for $v$ and $V$, since we have shown it to be a conformal invariant. Then $W=F\left[f^{-1}(w)\right] \equiv h(w)$ is a conformal map of the exterior of $\gamma_{1}$ onto the exterior of $\Gamma_{1}$, which takes $\delta^{\prime}$ onto $\Delta^{\prime}$. We will prove that this map is linear by showing that it can be extended to be one to one and conformal everywhere in the (extended) $w$-plane. 
Let $\gamma$ and $\Gamma$ be the images of $C$ in the $w$ and $W$-planes respectively. We see that $[v(w)-1] / A$ is the Green's function of the interior of $\gamma$ with pole at $w_{0}$, from which we may construct in the usual way a conformal map $t=g(w)$ of the interior of $\gamma$ onto the interior of the unit circle $|t|<1$ which sends $w_{0}$ onto $t=0$. Since $\gamma_{1}$ is a level locus of the Green's function, the image of $\delta^{\prime}$ under this map is the annulus $e^{-1 / A}<|t|<1$. By the identical argument, there exists a conformal map $T=G(W)$ of the interior of $\Gamma$ onto the interior of the unit circle $|T|<1$, by which $\Delta^{\prime}$ is mapped onto the annulus $e^{-1 / A}$ $<|T|<1$. The composed map $T=G\left\{h\left[g^{-1}(t)\right]\right\}$ now takes the above two annuli onto each other, with the unit circumferences corresponding; it is thus

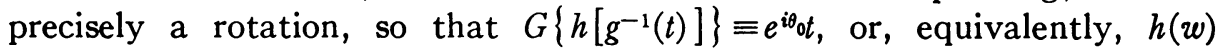
$\equiv G^{-1}\left[e^{i \theta_{0}} g(w)\right]$, for $w$ in $\delta^{\prime}$. But the map $W=G^{-1}\left[e^{i \theta_{0}} g(w)\right]$ is one to one and conformal not only on $\delta^{\prime}$ but everywhere interior to $\gamma$ as well; it therefore provides the required extension of $h(w)$ to the entire $w$-plane. Thus $W=h(w)$ is a one to one conformal map of the $w$-plane onto the $W$-plane; it is therefore a linear transformation as was to be proved. Theorem 1 is established.

Corollary 1. Let $D$ be a region of the z-plane, bounded by the contour $C$; to fix.the ideas, let $D$ be the exterior of $C$. Let $u(z)$ be a harmonic function defined in a neighborhood of $C$ in $D$, and suppose $u(z)$ to be continuous and constant on $C$ with no critical point on $C$. Then there exists a map $w=f(z)$ with $f(\infty)=\infty$, one to one and conformal in $D$, mapping it onto the exterior of a contour $\gamma$, and such that $v(w)=u\left[f^{-1}(w)\right]$, the transform of $u(z)$, is extendible harmonically to the interior of $\gamma$ with the exception of one point, where it has a logarithmic singularity.

Proof. Let $N \subset D$ be the domain of definition of $u(z)$. Suppose $u(z)=k$ on $C ; u(z)$ may be extended by reflection to be harmonic in a neighborhood of $C$, so that $\partial u / \partial n$ exists everywhere on $C$ and is continuous there. Since $u(z)$ has no critical points on $C$, we have $\partial u / \partial n \neq 0$ on $C$, so that $\partial u / \partial n$ is either positive or negative everywhere on $C$. Thus, inside a sufficiently small neighborhood of $C$, the values of $u(z)$ in $N$ are all either larger or smaller than $k$; suppose that they are larger. Let $C^{\prime}$ be a Jordan curve in $N$, such that $C^{\prime}$ and $C$ together bound an annular domain $R$ contained in the above neighborhood of $C$, and let $\alpha=\min _{z \in C^{\prime}} u(z)$. We have $\alpha>k$. Now select $\beta$ so that $\alpha>\beta>k$, and consider in $R$ the locus $\lambda: u(z)=\beta$. The locus $\lambda$ is a closed curve in $R$. It cannot be made up of more than one closed Jordan curve, since two closed curves in an annular domain must either be homotopic to each other, or have one of them homotopic to zero: in each case there would exist a subdomain of $R$ on whose boundary $u(z)=\beta$, which would imply that $u(z) \equiv \beta$ in $R$, contradicting the nature of $u(z)$. Consequently the locus $\lambda$ has no critical points of $u(z)$, else it would be the union of more than one closed Jordan curve, which was seen to be impossible; it is therefore an analytic Jordan curve which, together with $C$, bounds an annular domain $R^{\prime}$. The harmonic meas- 
ure of $R^{\prime}$ is seen to be $v(z) \equiv[u(z)-k] /(\beta-k)$, and by Theorem 1 there exists a one to one conformal map $w=f(z)$ of the exterior of $C$ onto the exterior of a contour $\gamma$, with $f(\infty)=\infty$, such that the transform $v\left[f^{-1}(w)\right]$ of $v(z)$ is extendible harmonically everywhere to the interior of $\gamma$ with the exception of a single point where it has a logarithmic singularity. Since

$$
v\left[f^{-1}(w)\right] \equiv \frac{u\left[f^{-1}(w)\right]-k}{\beta-k},
$$

we see that the transform $u\left[f^{-1}(w)\right]$ of $u(z)$ under the map $w=f(z)$ is also extendible harmonically everywhere to the interior of $\gamma$ with the exception of one point at which it has a logarithmic singularity.

If the values of $u(z)$ in $R$ are smaller than $k$, let $\alpha=\max _{z \in C}, u(z)$. We have $k>\alpha$, and selecting $\beta$ so that $k>\beta>\alpha$ we argue precisely as before. Corollary 1 is established.

TheOREM 2 (WALSh). Let $D$ be a region of the extended $z$-plane whose boundary consists of mutually disjoint Jordan curves $B_{1}, B_{2}, \cdots, B_{\mu} ; C_{1}, C_{2}$, $\cdots, C_{\nu}, \mu \nu \neq 0$. There exists a conformal map of $D$ onto a region $\Delta$ of the extended $Z$-plane, one to one and continuous in the closures of the two regions, where $\Delta$ is defined by

$$
1<|T(Z)|<e^{\alpha}, \quad T(Z) \equiv \frac{A\left(Z-a_{1}\right)^{M_{1}} \cdots\left(Z-a_{\mu}\right)^{M_{\mu}}}{\left(Z-b_{1}\right)^{N_{1}} \cdots\left(Z-b_{\nu}\right)^{N_{\nu}}},
$$

with $\alpha, M_{i}, N_{j}>0, \sum M_{i}=\sum N_{j}=1$. The locus $|T(Z)|=1$ consists of $\mu$ mutually disjoint Jordan curves $B_{i}^{*}$, respective images of the $B_{i}$, which separate $\Delta$ from the $a_{i}$ : the locus $|T(Z)|=e^{\alpha}$ consists of $\nu$ mutually disjoint Jordan curves $C_{j}^{*}$, respective images of the $C_{j}$, which separate $\Delta$ from the $b_{j}$.

Proof. Assume the Jordan curves $B_{1}, \cdots, B_{\mu} ; C_{1}, \cdots, C_{\nu}$ to be all analytic and $D$ to contain $z=\infty$, since that property may always be obtained by a preliminary conformal transformation of $D$. The harmonic measure $u(z)$ of the point set $\bigcup_{j=1}^{\nu} C_{j}$ with respect to the domain $D$ may be extended, by reflection across the analytic boundary curves, to be harmonic in a neighborhood of the boundary of $D$. The function $u(z)$ cannot have a critical point on the boundary of $D$, since a level locus of a harmonic function which passes through a critical point consists in a neighborhood of that point of $2 n$ (with $n>1$ ) analytic arcs, radiating from the critical point and spaced at equal angles $\pi / n$ one from the next; thus if a critical point lay on one of the boundary curves $B_{i}$ or $C_{j}$, there would be points interior to $D$ at which $u(z)$ had the values 1 or 0 , contradicting the maximum principle. By Corollary 1 , there exists a map $w_{1}=f_{1}(z)$, one to one and conformal exterior to $B_{1}$, with $f_{1}(\infty)$ $=\infty$, taking $D$ onto a domain $\Delta_{1}$ and $B_{1}$ onto a curve $\beta_{1}$, such that the function $u_{1}\left(w_{1}\right) \equiv u\left[f^{-1}\left(w_{1}\right)\right]$, which corresponds in the $w_{1}$-plane to $u(z)$, is extend- 
ible harmonically to the interior of $\beta_{1}$ with the exception of one point, where it has a logarithmic singularity.

The above procedure may next be applied to the image in the $w_{1}$-plane of $B_{\mathbf{2}}$, and so on successively for all the $B_{i}$ and $C_{j}$. Denote $w_{\mu+\nu}$ by $Z$; the combined effect of the $\mu+\nu$ appropriate successive maps is to take $D$ one to one and conformally onto a region $\Delta$ of the $Z$-plane, where the function which corresponds to $u(z)$ may be extended harmonically to the entire plane, with the exception of one point in each of the components of the complement of $\Delta$, where it has a logarithmic singularity. Consequently, $u(z) \equiv \log |T(z)| / \alpha$, with $T(Z)$ of the form (2), and $\Delta$ may be defined by $1<|T(Z)|<e^{\alpha}$. Theorem it 2 is established.

We show next that the canonical conformal map of Theorem 2 is unique up to a linear transformation of the $Z$-plane.

TheOREM 3 (WALSh). Let $D$ be a region of the z-plane defined by the inequality

$1<|R(z)|<e^{\alpha}, \quad R(z) \equiv \frac{A\left(z-a_{1}\right)^{m_{1}} \cdots\left(z-a_{\mu}\right)^{m_{\mu}}}{\left(z-b_{1}\right)^{n_{1}} \cdots\left(z-b_{\nu}\right)^{n_{\nu}}}, \quad \sum m_{i}=\sum n_{j}=1$,

and whose boundary consists of mutually disjoint Jordan curves $B_{1}, B_{2}, \cdots, B_{\mu}$; $C_{1}, C_{2}, \cdots, C_{\nu}$, where $B_{i}$ separates $a_{i}$ from $D$ and $C_{j}$ separates $b_{j}$ from $D$. Let $\Delta$ be a region of the $Z$-plane defined by

$$
\begin{gathered}
1<\left|R_{1}(Z)\right|<e^{\alpha^{\prime}} \quad R_{1}(Z) \equiv \frac{A^{\prime}\left(Z-a_{1}^{\prime}\right)^{m_{1}{ }^{\prime}} \cdots\left(Z-a_{\mu}^{\prime}\right)^{m_{\mu^{\prime}}}}{\left(Z-b_{1}^{\prime}\right)^{n_{1}{ }^{\prime}} \cdots\left(Z-b_{\nu}^{\prime}\right)^{n_{\nu^{\prime}}}}, \\
\sum m_{i}^{\prime}=\sum n_{j}^{\prime}=1,
\end{gathered}
$$

and whose boundary consists of mutually disjoint Jordan curves $B_{1}^{\prime}, B_{2}^{\prime}, \cdots, B_{\mu}^{\prime}$; $C_{1}^{\prime}, C_{2}^{\prime}, \cdots, C_{\nu}^{\prime}$, where $B_{i}^{\prime}$ separates $a_{i}^{\prime}$ from $\Delta$ and $C_{j}^{\prime}$ separates $b_{j}^{\prime}$ from $\Delta$. If there exists $a$ one to one conformal transformation $Z=f(z)$ of $D$ onto $\Delta$ so that each $B_{i}$ corresponds to $B_{i}^{\prime}$ and each $C_{j}$ corresponds to $C_{j}^{\prime}$, then the transformation is a linear transformation of the complex variable $z$, defined throughout the extended planes $z$ and $Z$. We have $\alpha=\alpha^{\prime}, m_{j}=m_{j}^{\prime}$, and $n_{j}=n_{j}^{\prime}$.

Proof. Let $u(z)$ be the harmonic measure of the point set $\bigcup_{j=1}^{\nu} C_{j}$ with respect to $D$, and let $U(Z)$ be the harmonic measure of the point set $\bigcup_{j=1}^{\nu} C_{j}^{\prime}$ with respect to $\Delta$. By definition of the two domains, $u(z) \equiv \log |R(z)| / \alpha$ and $U(Z) \equiv \log \left|R_{1}(Z)\right|<\alpha^{\prime}$, so that the two harmonic measures are extendible harmonically everywhere to the $z$ and $Z$-planes, except for one point in each component of the complements of $D$ and $\Delta$, where they have logarithmic singularities. As in the proof of Theorem 2, the function $u(z)$ cannot have a critical point on the boundary of $D$. Therefore, applying the proof of Corollary 1 to the boundary contour $B_{1}$, we may find a contour $\gamma$ in $D$ on which $u(z)$ is constant and which, together with $B_{1}$, bounds an annular domain $D^{\prime}$. 
On $\Delta$ we have $U(Z) \equiv u\left[f^{-1}(z)\right]$, since both functions are harmonic in $\Delta$ and assume the same values on the boundary of $\Delta$; it follows that the image $\Gamma$ of $\gamma$ under $Z=f(z)$ is a contour in $\Delta$ on which $U(Z)$ has the same constant value and which, together with $B_{1}^{\prime}$, bounds an annular domain $\Delta^{\prime}$. Now proceeding precisely as in the uniqueness proof of Theorem 1 , we may extend the map $Z=f(z)$ to be one to one and conformal in the interior of $\gamma$, with $f\left(a_{1}\right)=a_{1}^{\prime}$.

Applying the identical argument to the remaining boundary contours of $D$, we see that the map $Z=f(z)$ is extendible to a one to one conformal map of the extended $z$-plane onto the extended $Z$-plane, and is therefore a linear transformation. By the construction, $Z=f(z)$ sends the points $z=a_{i}$ onto $Z=a_{i}^{\prime}$ and $z=b_{j}$ onto $Z=b_{j}^{\prime}$. The singularities of $u(z)$ at $a_{i}$ and $b_{j}$ are given by $m_{i} \log \left|z-a_{i}\right| / \alpha$ and $-n_{j} \log \left|z-b_{j}\right| / \alpha$ respectively; analogously for $U(Z)$. Since $u(z)$ and $U(Z)$ are transforms of each other under the linear map, they have the same singularities, so that $m_{i} / \alpha=m_{i}^{\prime} / \alpha^{\prime}$ and $n_{j} / \alpha=n_{j}^{\prime} / \alpha^{\prime}$. Adding these equalities we conclude that $\alpha=\alpha^{\prime}, m_{i}=m_{i}^{\prime}$, and $n_{j}=n_{j}^{\prime}$. Theorem 3 is established.

TheOREM 4 (WALSH). Let $D$ be a region of the Z-plane whose boundary consists of mutually disjoint Jordan curves $C_{1}, \cdots, C_{\nu}$, let $\alpha_{1}, \cdots, \alpha_{\mu}$ be arbitrary distinct points of $D$, and let $M_{1}, \cdots, M_{\mu}$ be arbitrary positive numbers with $\sum M_{i}=1$. Then there exists a conformal map of $D$ onto a region $\Delta$ of the $Z$-plane, one to one and continuous in the closed regions, where $\Delta$ is defined by

$$
|T(Z)|<1, T(Z) \equiv \frac{A\left(Z-a_{1}\right)^{M_{1}} \cdots\left(Z-a_{\mu}\right)^{M_{\mu}}}{\left(Z-b_{1}\right)^{N_{1}} \cdots\left(Z-b_{\nu}\right)^{N_{\nu}}}, N_{j}>0, \sum N_{j}=1 .
$$

The $a_{i}$ are the respective images of the $\alpha_{i}$; the locus $|T(Z)|=1$ consists of $\nu$ analytic Jordan curves, respective images of the $C_{j}$, which separate $\Delta$ from the $b_{j}$.

Proof. Theorem 3 represents the limiting case of Theorem 2 as the curves $B_{i}$ shrink to points. It may be established by applying without change the argument above to the function $u(z)$ which is harmonic in $D$ except for singularities $M_{i} \log \left|z-\alpha_{i}\right|$ at the points $z=\alpha_{i}$, and which takes on the value zero on $\bigcup_{j=1}^{\nu} C_{j}$.

The map of Theorem 4 is likewise uniquely determined, except for a possible linear transformation.

Theorem 5 (WALsh). Let $D$ be a region of the z-plane defined by the inequality

$|R(z)|<1, R(z) \equiv \frac{A\left(z-a_{1}\right)^{m_{1}} \cdots\left(z-a_{\mu}\right)^{m_{\mu}}}{\left(z-b_{1}\right)^{n_{1}} \cdots\left(z-b_{\nu}\right)^{n_{\nu^{\prime}}}}, m_{i}>0, \sum m_{i}=\sum n_{j}=1$,

and whose boundary consists of mutually disjoint Jordan curves $C_{1}, C_{2}, \cdots, C_{\nu}$, which separate the $b_{j}$ respectively from $D$. Let $\Delta$ be a region of the $Z$-plane defined by the inequality 


$$
\left|R_{1}(Z)\right|<1, \quad R_{1}(Z) \equiv \frac{A^{\prime}\left(Z-a_{1}^{\prime}\right)^{m_{1}} \cdots\left(Z-a_{\mu}^{\prime}\right)^{m_{\mu}}}{\left(Z-b_{1}^{\prime}\right)^{n_{1}^{\prime}} \cdots\left(Z-b_{v}^{\prime}\right)^{n^{\prime}{ }_{p}}}, \quad \sum n_{j}^{\prime}=1,
$$

and whose boundary consists of mutually disjoint Jordan curves $C_{1}^{\prime}, C_{2}^{\prime}, \cdots, C_{\nu}^{\prime}$, which separate the $b_{j}^{\prime}$ respectively from $\Delta$. If there exists a one to one conformal transformation of $D$ onto $\Delta$ so that the $a_{i}$ correspond respectively to the $a_{i}^{\prime}$, then this correspondence can be continued beyond $D$ and $\Delta$ as a linear transformation of the extended z-plane onto the extended $Z$-plane, and $n_{j}=n_{j}^{\prime}$.

Proof. We establish Theorem 5 by applying without change the argument of Theorem 3, to the functions $u(z)$ and $U(Z)$ which are harmonic in $D$ and $\Delta$ except for singularities $m_{i} \log \left|z-a_{i}\right|$ and $m_{i} \log \left|Z-a_{i}^{\prime}\right|$ at the points $z=a_{i}$ and $Z=a_{i}^{\prime}$ respectively and which take on the value zero on $\bigcup_{j=1}^{\nu} C_{j}$ and $\bigcup_{j=1}^{\nu} C_{j}^{\prime}$.

II. The canonical map and extremal problems. We will now consider the relation between the canonical maps of Theorems 2 and 4 and extremal problems.

The conformal map $Z=f(z)$ of a doubly connected domain $D$ onto an annulus $r_{1}<|Z|<r_{2}$ may, by a simple application of Schwarz's Lemma, be seen to possess the following extremal characterization: let $Z=g(z)$ be any univalent map of $D$ onto a bounded doubly connected domain $D_{\theta}$ in the $Z$ plane whose inner and outer boundaries are Jordan curves $B_{g}$ and $C_{g}$ respectively, with $Z=0$ interior to $B_{0}$. Set $M_{0}=\min _{Z \in B_{0}}|Z| / \max _{z \in C_{0}}|Z|$; then the map $Z=f(z)$ has the property that

$$
M_{f} \geqq M_{g} \text {. }
$$

This extremal property may be viewed somewhat differently. Consider the family $H$ of functions $h(z)$ harmonic in $D$, continuous in the closure $\bar{D}$ of $D$, and normalized so that $\int_{\gamma} \partial h / \partial n d s=2 \pi$, where $\gamma$ is an analytic Jordan curve in $D$ which separates the boundary curves $B$ and $C$ of $D$, and $n$ is the normal on $\gamma$ in the direction of $C$. For $h(z) \in H$, let $R_{h}=\max _{z \in \bar{D}} h(z)$ $-\min _{z \in \bar{D}} h(z)$, and consider the problem of minimizing $R_{h}$ over the family $H$. We will show (Corollary 2) that $R_{h}$ is minimized in $H$ by any function of the form $\alpha u(z)+k$, where $u(z)$ is the harmonic measure of the curve $C$ with respect to $D$, the normalizing constant $\alpha=2 \pi / \int_{\gamma} \partial u / \partial n d s$, and $k$ is an arbitrary constant. This extremal problem immediately induces one among the univalent functions of $D$. For every one of the univalent maps $Z=g(z)$ of $D$ defined above, the function $\log |Z| \equiv \log |g(z)|$ is contained in $H$, by virtue of the normalization of the maps, while the function $\log |f(z)|$ coincides with the particular extremal function $\alpha u(z)+\log r_{1}$. Thus $R_{\log |f|} \leqq R_{\log |\rho|}$, or

$$
\frac{\max _{z \in \bar{D}}|f(z)|}{\min _{z \in \bar{D}}|f(z)|} \leqq \frac{\max _{z \in D}|g(z)|}{\min _{z \in \bar{D}}|g(z)|} .
$$


But by virtue of the assumption that $B_{0}$ and $C_{0}$ are the inner and outer boundary curves of $D_{g}$, the quantities $\max _{z \in \bar{D}}|g|$ and $\min _{z \in \bar{D}}|g|$ coincide respectively with $\max _{Z \in C_{0}}|g|$ and $\min _{Z \in B_{o}}|g|$, so that (5) is equivalent to the inequality (4) above.

The analogous formulation may be given for the canonical map $Z=f(z)$ in the domain $D$ of Theorem 2. We first consider the family $H$ of functions $h(z)$ harmonic in $D$, continuous in the closure $\bar{D}$ of $D$, and so normalized that $\int_{\gamma} \partial h / \partial n d s=2 \pi$, where $\gamma$ is an analytic Jordan curve in $D$ separating the curves $\bigcup_{i=1}^{\mu} B_{i}$ from the curves $\bigcup_{j=1}^{\nu} C_{j}$. Defining $R_{h}$ as above, we will show (Corollary 2) that $R_{h}$ is again minimized in $H$ by any function of the form $\alpha u(z)+k$, where $u(z)$ is the harmonic measure of the curves $U_{j=1}^{\nu} C_{j}$ with respect to $D$, the normalizing constant $\alpha=2 \pi / \int_{\gamma} \partial u / \partial n d s$, and $k$ is an arbitrary constant. Since the function $\log |T[f(z)]|$, where $Z=f(z)$ and $T(Z)$ are as in Theorem 2, coincides with the specific extremal function $\alpha u(z)$, we have in particular $R_{\log |T(f)|} \leqq R_{h}$. From this we will below obtain an inequality which refers explicitly to univalent maps of $D$ and whose form is modeled after (4).

THEOREM 6. Let $D$ be a region of the extended z-plane whose boundary consists of mutually disjoint Jordan curves $B_{1}, \cdots, B_{\mu} ; C_{1}, \cdots, C_{\nu}, \mu \nu \neq 0$. Let $u(z)$ be the harmonic measure of the set $\bigcup_{j=1}^{\nu} C_{j}$ with respect to the domain $D$, and let $v(z)$ be any function harmonic in $D$, continuous in the closure $\bar{D}$ of $D$, and such that $v(z) \geqq 0$ on $\bigcup_{i=1}^{\mu} B_{i}$ and $v(z) \leqq 1$ on $\bigcup_{j=1}^{\nu} C_{j}$. Let $\gamma$ be an analytic Jordan curve in $D$ which separates the curves $\bigcup_{i=1}^{\mu} B_{i}$ from the curves $\bigcup_{j=1}^{p} C_{j}$. Then

$$
\int_{\gamma} \partial u / \partial n d s \geqq \int_{\gamma} \partial v / \partial n d s,
$$

where $n$ is the normal on $\gamma$ in the direction of the curves $\bigcup_{j-1}^{y} C_{j}$. Equality occurs if and only if $v(z) \equiv u(z)$ in $\bar{D}$.

Proof. We may assume the curves $B_{i}$ and $C_{j}, i=1, \cdots, \mu ; j=1, \cdots, \nu$, to be all analytic Jordan curves, for this property may always be obtained by a preliminary conformal transformation of $D$. Let $p(z) \equiv u(z)-v(z)$; then $p(z)$ is harmonic in $D$, with $p(z) \leqq 0$ for $z$ in $\bigcup_{i=1}^{\mu} B_{i}$ and $p(z) \geqq 0$ for $z$ in $\bigcup_{j=1}^{\nu} C_{j}$. Let $q(z)$ be the function harmonic in $D$ which has value zero on $\bigcup_{j=1}^{y} C_{j}$ and which coincides with $p(z)$ on $\bigcup_{i=1}^{\mu} B_{i}$, and let $r(z) \equiv p(z)-q(z)$. Now $r(z)=0$ for $z$ in $\bigcup_{i=1}^{\mu} B_{i}$ and $r(z)=p(z) \geqq 0$ for $z$ in $\bigcup_{j=1}^{\nu} C_{j}$. The function $r(z)$ may be extended by reflection across the part of the boundary made up of the curves $B_{i}$ to be harmonic in a neighborhood of $\bigcup_{i=1}^{\mu} B_{i}$, so that the normal derivative of $r(z)$ exists almost everywhere on $\bigcup_{i=1}^{\mu} B_{i}$. By the maximum principle, $r(z) \geqq 0$ in $D$, so that $\partial r(z) / \partial n_{i} \geqq 0$ for $Z \in \bigcup_{i=1}^{\mu} B_{i}$, with $n_{i}$ the interior normal. Hence 


$$
0 \leqq \int_{\cup_{i} B_{i}} \partial r / \partial n_{i} d s=\int_{\gamma} \partial r / \partial n d s
$$

with $n$ the normal on $\gamma$ in the direction of the curves $\bigcup_{j=1}^{\nu} C_{j}$, or, from the definition of $r(z)$,

$$
\int_{\gamma} \partial p / \partial n d s \geqq \int_{\gamma} \partial q / \partial n d s
$$

Now, arguing as with $r(z)$, the function $q(z)$ may be extended to be harmonic in a neighborhood of the curves $\bigcup_{j=1}^{v} C_{j}$, so that its normal derivative exists almost everywhere on $\mathrm{U}_{j=1}^{\nu} C_{j}$. By the maximum principle, $q(z) \leqq 0$ in $D$, so that $\partial q(z) / \partial n_{e} \geqq 0$ for $z \in \bigcup_{j=1}^{\nu} C_{j}$, with $n_{e}$ the exterior normal. Thus

$$
0 \leqq \int_{\cup_{j} c_{j}} \partial q / \partial n_{e} d s=\int_{\gamma} \partial q / \partial n d s
$$

Combining (6) and (7) we obtain

$$
\int_{\gamma} \partial p / \partial n d s \geqq 0
$$

or

$$
\int_{\gamma} \partial u / \partial n d s \geqq \int_{\gamma} \partial v / \partial n d s
$$

If equality occurs in (8), by retracing the steps of the proof we see that we must have $\partial q / \partial n=0$ almost everywhere for $z \in \bigcup_{j=1}^{\nu} C_{j}$, and $\partial r / \partial n=0$ almost everywhere for $z \in \bigcup_{i=1}^{\mu} B_{i}$. The function $q(z)$ is harmonic in a neighborhood of the curves $C_{j}$ and has value 0 for $z \in \bigcup_{j=1}^{\nu} C_{j}$. Choose a point $z_{0}$ in $\mathrm{U}_{j=1}^{\nu} C_{j}$ and a simply connected neighborhood $N$ of $z_{0}$ such that $q(z)$ is harmonic in $N$, and let $q^{*}(z)$ be the conjugate harmonic function of $q(z)$ in $N$. On any $\operatorname{arc} A$ of $\bigcup_{j=1}^{y} C_{j}$ contained in $N$ we have $\partial q^{*}(z) / \partial s=\partial q(z) / \partial n=0$, so that the function $q(z)+i q^{*}(z)$, analytic in $N$, is constant on the $\operatorname{arc} A$ in $N$, hence identically constant in $N$. Thus $q(z) \equiv 0$ in $N$, whence $q(z) \equiv 0$ in $\bar{D}$. Arguing analogously, $r(z) \equiv 0$ in $\bar{D}$. But this implies that $p(z) \equiv 0$ for $z \in \cup_{i=1}^{\mu} B_{i}$ and $z \in \cup_{j=1}^{p} C_{j}$, thus also $p(z) \equiv 0$ in $\bar{D}$, whence $v(z) \equiv u(z)$ in $\bar{D}$. Theorem 6 is established.

CoRollary 2. Let $H$ be the family of functions $h(z)$ harmonic in $D$, continuous in the closure $\bar{D}$ of $D$, and normalized so that $\int_{\gamma} \partial h / \partial n d s=2 \pi$, where $\gamma$ is an analytic Jordan curve in $D$ separating the curves $\bigcup_{i=1}^{\mu} B_{i}$ from the curves $\bigcup_{i=1}^{\nu} C_{j}$, and $n$ is the normal on $\gamma$ in the direction of $\bigcup_{j=1}^{\nu} C_{j}$. Let $R_{h}=\max _{z \in \bar{D}} h(z)$ $-\min _{z \in \bar{D}} h(z)$, and let $w(z) \equiv 2 \pi u(z) / \int_{\gamma} \partial u / \partial n d s$ with $u(z)$, as before, being the 
harmonic measure of the curves $\bigcup_{j=1}^{\nu} C_{j}$ with respect to $D$. Then $R_{w} \leqq R_{h}$, for all $h(z) \in H$, with equality if and only if $h(z) \equiv w(z)+k, k$ a constant.

Proof. If $h(z) \in H$, the quantity $R_{h} \neq 0$, else $h(z) \equiv c$ in $D$, which contradicts the normalization requirement. Consider then, for every $h(z) \in H$, the function $h^{*}(z)=\left[h(z)-\min _{z \in \bar{D}} h(z)\right] / R_{h}$; we have $0 \leqq h^{*} \leqq 1$ in $\bar{D}$, so that, by Theorem 6 ,

$$
\int_{\gamma} \partial u / \partial n d s \geqq \int_{\gamma} \partial h^{*} / \partial n d s=\int_{\gamma} \partial h / \partial n d s / R_{h}=2 \pi / R_{h} .
$$

Now $w(z)$ has constant boundary values, 0 and $2 \pi / \int_{\gamma} \partial u / \partial n d s$ respectively, on the boundary of $D$, and, as in Theorem 6, the latter quantity is positive. Thus $R_{w}=2 \pi / \int_{\gamma} \partial u / \partial n d s$ or, from (9), $2 \pi / R_{w} \geqq 2 \pi / R_{h}$, whence $R_{w} \leqq R_{h}$. Equality occurs if and only if it occurs in (9), that is, if and only if $h^{*}(z) \equiv u(z)$ (Theorem 6). By definition this is equivalent to $h(z)-\min _{z \in \bar{D}} h(z) \equiv R_{h} u(z)$ $\equiv w(z)$, whence $h(z) \equiv w(z)+\min _{z \in \bar{D}} h(z)$. Corollary 2 is established.

We can now specialize the above extremal problem to the univalent maps of $D$, by restricting consideration to the subfamily of $H$ consisting of only those functions which are each extendible harmonically everywhere to the complement, except for points, one in each of the components of the complement, of the image of $D$ under some univalent map. Let $Z=g(z)$ be any univalent map of $D$ onto a domain $D_{g}$ of the $Z$-plane, bounded by Jordan curves $\bigcup_{i-1}^{\mu} B_{i, \theta}$ and $\bigcup_{j=1}^{v} C_{j, \theta}$, with $B_{i, \theta}$ and $C_{j, \theta}$ the images of $B_{i}$ and $C_{j}$ respectively under the map $Z=g(z)$. Let $S_{o}(Z)$ be any function of the form

$$
S_{0}(Z) \equiv \frac{C\left(Z-\alpha_{1}\right)^{s_{1}} \cdots\left(Z-\alpha_{\mu}\right)^{s_{\mu}}}{\left(Z-\beta_{1}\right)^{t_{1}} \cdots\left(Z-\beta_{v}\right)^{t_{\nu}}}
$$

where $s_{i}, t_{j}>0, \sum s_{i}=\sum t_{j}=1$, and $\alpha_{i}$ and $\beta_{j}$ are separated from $D_{0}$ by the curves $B_{i, \theta}$ and $C_{j, \theta}$ respectively. Since $S_{\theta}(Z)$ has neither zeros nor poles in the closure $\bar{D}_{o}$ of $D_{\theta}$, the functions $\log \left|S_{\theta}(Z)\right| \equiv \log \left|S_{o}[g(z)]\right|$ are all harmonic in $D$ and continuous in the closure of $D$. If $\gamma$ is as in Corollary 2, the image $\Gamma$ of $\gamma$ under the map $Z=g(z)$ separates the curves $\cup_{i-1}^{\mu} B_{i, 0}$ from the curves $\bigcup_{j=1}^{v} C_{j, 0}$ in $D_{o}$, so that, from the form of $S_{o}(Z)$, we have that $\left[\arg S_{o}(Z)\right]_{\Gamma}$ $=2 \pi$. But

$$
\left[\arg S_{o}(Z)\right]_{\Gamma}=\int_{\Gamma} \partial \arg \left[S_{o}(Z)\right] / \partial s d s=\int_{\Gamma} \partial \log \left|S_{o}(Z)\right| / \partial n d s,
$$

so that functions $\log \left|S_{0}(Z)\right|$ satisfy the normalization condition of Corollary 2 as well, and hence are all in $H$. The function $\log \left|T_{f}(Z)\right|=\log |T[f(z)]|$, where $Z=f(z)$ and $T(Z)$ are as in Theorem 2, is in $H$ and has boundary values 0 on the curves $\bigcup_{i=1}^{\mu} B_{i}$ and $\alpha$ on the curves $\bigcup_{j=1}^{p} C_{j}$, so that it coincides with the extremal function $w(z)$ of Corollary 2 . Thus, by Corollary $2, R_{\log |T[f]|}$ $\leqq R_{\log \left|S_{0}[0]\right|}$, or 


$$
\frac{\max _{z \in \bar{D}} \log |T[f(z)]|}{\min _{z \in \bar{D}} \log |T[f(z)]|} \leqq \frac{\max _{z \in \bar{D}} \log \left|S_{o}[g(z)]\right|}{\min _{z \in \bar{D}} \log \left|S_{o}[g(z)]\right|} .
$$

The functions $\log \left|S_{\vartheta}(Z)\right|$ are all harmonic on the respective domains $D_{0}$ and hence assume their maxima and minima on the boundary of $D_{0}$. Any level locus $\log \left|S_{g}(Z)\right|=c$ is composed of a finite number of closed Jordan curves and separates points in the $Z$-plane where $\log \left|S_{o}(Z)\right|>c$ from points where $\log \left|S_{o}(Z)\right|<c$. Furthermore, every region bounded by curves of this locus must contain in its interior points of singularity of the function $\log \left|S_{g}(Z)\right|$, that is, one or more of the points $\alpha_{i}$ or $\beta_{j}$, else the function would have constant boundary values in a region of harmonicity which implies that it is identically constant, contrary to its form (10). Let $\lambda=\max _{Z \in \bar{D}_{g}} \log \left|S_{g}(Z)\right|$ $=\max _{\mathbf{z} \in \bar{D}} \log \left|S_{0}[g(z)]\right|$. The level $\operatorname{locus} \log \left|S_{0}(Z)\right|=\lambda$ lies entirely in the complement of $D_{0}$. No Jordan curve $J$ of this locus can lie inside a component of the complement of $D_{\theta}$ bounded by one of the curves $B_{i, g}$ since $J$ would then separate $D_{o}$ from $Z=\alpha_{i}$, hence also from points where $\log \left|S_{o}(Z)\right|<\lambda$; this would imply that $\log \left|S_{\theta}(Z)\right|>\lambda$ on $D_{\theta}$, contradicting the definition of $\lambda$. Consequently the function $\log \left|S_{o}(Z)\right|$ assumes its maximum in $\bar{D}_{g}$ on the boundary curves $\bigcup_{j=1}^{y} C_{j, o}$; by the identical argument, $\min _{z \in \bar{D}} \log \left|S_{o}[g(z)]\right|$ $=\min _{z \in \cup_{i} B_{i}} \log \left|S_{o}[g(z)]\right|$. We may therefore replace (11) by

$$
\frac{\max _{z \in \cup_{j} C_{j}} \log |T[f(z)]|}{\min _{z \in \cup_{i} B_{i}} \log |T[f(z)]|} \leqq \frac{\max _{z \in \cup_{j} C_{j}} \log \left|S_{o}[g(z)]\right|}{\min _{z \in \cup_{j} C_{j}} \log \left|S_{o}[g(z)]\right|} .
$$

Equality occurs in (12) if and only if it occurs in (11), that is, if and only if (Corollary 2) $\log \left|S_{0}(g)\right| \equiv \log |T(f)|+k$ for $z$ in $D$. If $\Delta$ is the canonical domain of Theorem 2: $1<\left|T\left(Z^{*}\right)\right|<e^{\alpha}$ which corresponds to $D$ in the $Z^{*}$ plane, with $T\left(Z^{*}\right)$ and $Z^{*}=f(z)$ as in Theorem 2, the map $Z=g\left[f^{-1}\left(Z^{*}\right)\right]$ provides a one to one conformal transformation of $\Delta$ onto $D_{\sigma}$. Furthermore, since $\left|e^{-k} S_{0}(g)\right| \equiv|T(f)|$, the above transformation maps the boundary curves $B_{i, \theta}$ and $C_{j, \theta}$ of $D_{0}$ onto the boundary curves $B_{i}^{*}$ and $C_{j}^{*}$ of $\Delta$ respectively, $i=1, \cdots, \mu ; j=1, \cdots, \nu$, and the domain $D_{0}$ is defined by $1<\left|e^{-k} S_{o}(Z)\right|<e^{\alpha}$. Theorem 3 may now be applied; it establishes that the map $Z=g\left[f^{-1}\left(Z^{*}\right)\right]$ is a linear transformation $Z=A\left(Z^{*}\right)$ of the complex variable $Z^{*}$, defined throughout the extended planes $Z$ and $Z^{*}$, or, equivalently, that $g(z)=A[f(z)]$. We have thus obtained in (12) an extremal statement modeled in form after (4) which singles out the canonical conformal map of Theorem 2 from among the univalent maps of $D$.

We turn next to a brief consideration of the number $\alpha$ which figures in Theorem 2; we will show it to be interpretable as an extremal length. The canonical domain associated with $D$ is the domain $1<|T(Z)|<e^{\alpha}$, where 
$Z=f(z)$ and $T(Z)$ are as defined in Theorem 2. As we have already shown above, the function $\log |T[f(z)]|$ is contained in the family $H$ of Corollary 2 , and coincides there with the function $\alpha u(z)$, where $u(z)$ is the harmonic measure of the curves $\bigcup_{j=1}^{\nu} C_{j}$ with respect to $D$. We thus have $\alpha=2 \pi / \int_{\gamma} \partial u / \partial n d s$, whence $\alpha$ is a conformal invariant. Furthermore

$$
\int_{\gamma} \partial u / \partial n d s=\int_{\cup_{j} C_{j}} \partial u / \partial n d s=\int_{\cup_{i} B_{i}+\cup_{j} C_{j}} u \partial u / \partial n d s,
$$

with $n$ the exterior normal, and by Green's theorem

$$
\int_{\cup_{i} B_{i}+\cup, C_{j}} u \partial u / \partial n d s=\iint_{D}(\partial u / \partial x)^{2}+(\partial u / \partial y)^{2} d x d y .
$$

Hence $\alpha / 2 \pi=1 / \iint_{D}\left(u_{x}\right)^{2}+\left(u_{y}\right)^{2} d x d y$, which is equal to the extremal length of the family of curves in $D$ joining points of $U_{i-1}^{\mu} B_{i}$ to points of $U_{j-1}^{p} C_{j}$ [2]; as such, $\alpha$ represents a natural generalization of the modulus of a doubly connected domain. The relation of the number $\alpha$ to the geometry of the domain $D$ may now be studied by using tools such as the Comparison and Composition Laws which apply to extremal length. In particular, $\alpha$ is a monotonic domain functional in the following sense.

Corollary 3. Let $D$ be the domain of Theorem 2. Let $D^{\prime}$ be a domain bounded by disjoint Jordan curves $B_{1}^{\prime}, \cdots, B_{m}^{\prime}$ and $C_{1}^{\prime}, \cdots, C_{n}^{\prime}, m n \neq 0$, such that $D^{\prime} \subset D$ and the sets of curves $\cup_{i=1}^{m} B_{i}^{\prime}$ and $\cup_{j=1}^{n} C_{j}^{\prime}$ each separate the curves $\bigcup_{i=1}^{\mu} B_{i}$ from the curves $\bigcup_{j=1}^{j} C_{j}$; that is, the sets of curves $\bigcup_{i=1}^{m} B_{i}^{\prime}$ and $\bigcup_{i=1}^{\mu} B_{i}$ together form the total boundary of a union of subregions of $D$. Let $\alpha^{\prime}$ be the constant corresponding to $\alpha$ in the definition of the canonical domain of $D^{\prime}$. Then $\alpha^{\prime} \leqq \alpha$.

Proof. The proof is immediate from the theory of extremal length $[1 ; 2 ; 3]$. The number $\alpha$ is equal to the extremal length of the family $F$ of curves in $D$ joining points of $\bigcup_{i=1}^{\mu} B_{i}$ to points of $U_{j=1}^{\nu} C_{j}$. Similarly, $\alpha^{\prime}$ is the extremal length of the family $F^{\prime}$ of curves in $D^{\prime}$ joining points of $\bigcup_{i=1}^{m} B_{i}^{\prime}$ to points of $\bigcup_{j=1}^{n} C_{j}^{\prime}$. By the Comparison Law applied to the families $F$ and $F^{\prime}$, we have $\alpha^{\prime} \leqq \alpha$. Corollary 3 may also be established without recourse to the theory of extremal length, by applying the argument of Theorem 6 .

We turn next to the canonical conformal map of Theorem 4 ; we will show that it is the solution of an extremal problem which is a generalization of the one solved by the map of a simply connected domain onto the interior of the unit circle.

Theorem 7. Let $Z=f(z)$ be the canonical map of Theorem 4 , taking $D$ onto a region of the $Z$-plane defined by $|T(Z)|<1$, with $T(Z)$ as in (3). Let $Z=g(z)$ be any one to one conformal map of $D$ onto a domain $D_{0}$ in the $Z$-plane bounded by Jordan curves, and let $S_{o}(Z)$ be any function of the form 


$$
S_{0}(Z) \equiv \frac{C\left(Z-c_{1}\right)^{M_{1}} \cdots\left(Z-c_{\mu}\right)^{M_{\mu}}}{\left(Z-d_{1}\right)^{t_{1}} \cdots\left(Z-d_{v}\right)^{t_{\nu}}},
$$

where $t_{j}>0, \sum t_{j}=1, c_{i}=g\left(\alpha_{i}\right)$, the point $Z=d_{j}$ is separated from $D_{0}$ by the image of $C_{j}$ under the map $Z=g(z)$ and such that

$$
\left|S_{\vartheta}(Z)\right|<1, \quad Z \in D_{0} \text {. }
$$

Let $F(z) \equiv T[f(z)]$, and $G(z) \equiv S_{o}[g(z)] ;$ let $p_{i}(z)=F(z) /\left[f(z)-a_{i}\right]^{M_{i}}$ and $q_{i}(z) \equiv G(z) /\left[g(z)-c_{i}\right]^{M_{i}}$. Then

$$
\left|f^{\prime}\left(\alpha_{i}\right)^{M_{i} p_{i}}\left(\alpha_{i}\right)\right| \geqq\left|g^{\prime}\left(\alpha_{i}\right)^{M_{i}} q_{i}\left(\alpha_{i}\right)\right|, \quad i=1, \cdots, \mu,
$$

with equality attained for any $i$ if and only if

$$
g(z) \equiv A[f(z)]
$$

with $A$ a linear transformation.

Proof. Consider for $z$ in $D$ the function $u(z) \equiv \log |F(z)|-\log |G(z)|$. The function $u(z)$ is harmonic for all $z$ in $D$ with the possible exception of the points $z=\alpha_{i}$, where $F(z)=G(z)=0$. In a neighborhood of $z=\alpha_{i}$, however, $p_{i}(z)$ and $q_{i}(z)$ are analytic $\neq 0$, and consequently

$$
\begin{aligned}
\log |F(z)| & =M_{i} \log \left|f(z)-a_{i}\right|+\log \left|p_{i}(z)\right| \\
& =M_{i} \log \left|z-\alpha_{i}\right|+M_{i} \log \left|f^{\prime}\left(\alpha_{i}\right)\right|+\log \left|p_{i}\left(\alpha_{i}\right)\right|+\delta_{i}(z),
\end{aligned}
$$

where $\delta_{i}(z)$ is harmonic in the neighborhood of $z=\alpha_{i}$ and $\lim _{z \rightarrow \alpha_{i}} \delta_{i}(z)=0$. Similarly, in a neighborhood of $z=\alpha_{i}$,

$$
\begin{aligned}
\log |G(z)| & =M_{i} \log \left|g(z)-c_{i}\right|+\log \left|q_{i}(z)\right| \\
& =M_{i} \log \left|z-\alpha_{i}\right|+M_{i} \log \left|g^{\prime}\left(\alpha_{i}\right)\right|+\log \left|q_{i}\left(\alpha_{i}\right)\right|+\epsilon_{i}(z),
\end{aligned}
$$

where $\epsilon_{i}(z)$ is harmonic in the neighborhood of $z=\alpha_{i}$ and $\lim _{z \rightarrow \alpha_{i}} \epsilon_{i}(z)=0$. Thus the function $u(z)$ has only a removable singularity at $z=\alpha_{i}$, and

$$
u\left(\alpha_{i}\right)=\log \left|f^{\prime}\left(\alpha_{i}\right)^{M_{i}} p_{i}\left(\alpha_{i}\right)\right|-\log \left|g^{\prime}\left(\alpha_{i}\right)^{M_{i}} q_{i}\left(\alpha_{i}\right)\right| .
$$

For $z \in \mathrm{U}_{j=1}^{y} C_{j}$ we have $\log |F(z)|=0$ and $\log |G(z)| \leqq 0$, thus $u(z) \geqq 0$. By the maximum principle, $u(z) \geqq 0$ at every interior point of $D$, with equality at any interior point only if $u(z) \equiv 0$ for $z$ in $D$. Hence, $u\left(\alpha_{i}\right) \geqq 0$, or

$$
\left|f^{\prime}\left(\alpha_{i}\right)^{M_{i}} p_{i}\left(\alpha_{i}\right)\right| \geqq\left|g^{\prime}\left(\alpha_{i}\right)^{M_{i}} q_{i}\left(\alpha_{i}\right)\right|
$$

for $i=1, \cdots, \mu$. If equality occurs for any $i$, we have $|F(z)| \equiv|G(z)|$, for $z$ in $D$. Arguing as in the sequel to Corollary 2 , we let $Z^{*}=f(z)$ be the canonical map of Theorem 4, taking $D$ onto the canonical domain $\Delta:\left|T\left(Z^{*}\right)\right|<1$ (with $T\left(Z^{*}\right)$ as in Theorem 4), and we consider the map $Z=g\left[f^{-1}\left(Z^{*}\right)\right]$ which provides a one to one conformal transformation of $\Delta$ onto $D_{g}$. Since $|F(z)| \equiv|G(z)|$, the above transformation maps the points $a_{i}$ onto the points 
$c_{i}$, and the domain $D_{0}$ is defined by $\left|S_{o}(Z)\right|<1$. Theorem 5 may now be applied to establish that the map $Z=g\left[f^{-1}\left(Z^{*}\right)\right]$ is a linear transformation $Z=A\left(Z^{*}\right)$ of the complex variable $Z^{*}$, defined throughout the extended $Z$ and $Z^{*}$-planes, or, equivalently, that $g(z) \equiv A[f(z)]$. Theorem 7 is established.

For the case that only a single point $\alpha_{i}$ is selected from $D$, we have $\mu=1$, and $M_{1}=1$; the extremal condition of Theorem 7 becomes

$$
\left|f^{\prime}\left(\alpha_{1}\right) p_{1}\left(\alpha_{1}\right)\right| \geqq\left|g^{\prime}\left(\alpha_{1}\right) q_{1}\left(\alpha_{1}\right)\right| \text {. }
$$

But $\left|f^{\prime}\left(\alpha_{1}\right) p_{1}\left(\alpha_{1}\right)\right|=\left|F^{\prime}\left(\alpha_{1}\right)\right|$ and $\left|g^{\prime}\left(\alpha_{1}\right) q_{1}\left(\alpha_{1}\right)\right|=\left|G^{\prime}\left(\alpha_{1}\right)\right|$. Thus the extremal condition which characterizes the canonical mapping function for the above special case of Theorem 4 is $\left|F^{\prime}\left(\alpha_{1}\right)\right|=\max _{G}\left|G^{\prime}\left(\alpha_{1}\right)\right|$. This extremal problem is a generalization of that which determines the mapping of a simply connected domain onto the interior of the unit circle.

III. Mapping a doubly connected domain onto an annulus. We show next how the construction of Theorem 1 may be applied to a doubly connected region $D$ to yield a rapidly converging method for performing the map of $D$ onto an annulus. A construction by successive approximations commonly used at present has been suggested by Y. Komatu [8] and consists of mapping $D$ onto doubly connected regions which are obtained each from the preceding by mapping alternately, and with appropriate normalization, the interiors of their outer and exteriors of their inner boundaries onto interiors and exteriors of circles, respectively. The rapidity of convergence of these approximations has been studied by D. Gaier [5], who has shown that $\left|F(z)-f_{n}(z)\right|<M_{1} \rho_{1}^{n}$, where $w=F(z)$ is a conformal map of $D$ onto an annulus, $w=f_{n}(z)$ is the approximation of Komatu constructed by means of $n$ conformal transformations of simply connected domains, and $M_{1}$ and $\rho_{1}<1$ are constants depending only on $D$. We will, below, define maps $w=F_{n}(z)$ of $D$, likewise obtained by means of $n$ conformal transformations of simply connected domains, and will demonstrate that $\left|F(z)-F_{n}(z)\right|<M \rho^{2^{n}}$, where $M$ and $\rho<1$ are constants depending only on $D$.

Let us again adopt the convention of using the term "harmonic measure of a doubly connected domain" to mean the harmonic measure of the outer boundary curve with respect to the domain.

Consider a doubly connected domain $D$ in the $z$-plane with outer contour $C$ and inner contour $C_{1}$. We may assume that $C$ is the unit circumference and $C_{1}$ surrounds $z=0$, for $D$ may always be mapped onto such a domain by a preliminary conformal transformation. Let $C^{*}$ be the reflection of $C_{1}$ in $C$; the harmonic measrure of $D$ is extendible by reflection to the domain $D^{*}$, bounded by $C_{1}$ and $C^{*}$, and has the value 2 on $C^{*}$.

Now let $Z=f(z)$ be the function obtained in Theorem 1 which is one to one and conformal in the entire exterior of $C_{1}$, with $f(\infty)=\infty$, and which maps $C_{1}$ onto some contour $\gamma_{1}$ and $D$ onto a domain $\Delta$ whose harmonic measure is extendible harmonically to the interior of $\boldsymbol{\gamma}_{1}$, except for the single point 
$Z=0$, where it has a logarithmic singularity. Let $\gamma$ and $\gamma^{*}$ be the images under $Z=f(z)$ of $C$ and $C^{*}$ respectively.

PROPOSITION 1. Let $w=h(Z)$ be the conformal map of the interior of $\gamma^{*}$ onto $|w|<1$, with $h(0)=0$, and $h^{\prime}(0)>0$. Then the map $w=h(Z)$ takes $\Delta$ onto an annulus in the w-plane.

Proof. Let $U(Z)$ be the harmonic measure of $\Delta$. By definition of $\Delta$, and as in the uniqueness proof of Theorem 1, the Green's function log $|h(Z)|$ of the interior of $\gamma^{*}$ with pole at $Z=0$ coincides with a constant multiple of $[U(Z)-2]$. Since the contours $\gamma$ and $\gamma_{1}$ are level loci of the latter function, the image of $\Delta$ under $w=h(Z)$ is an annulus. Proposition 1 is established.

Thus we see that in order to effect the map of $D$ onto an annulus, the map $Z=f(z)$ of Theorem 1 need be followed by only a single additional conformal transformation. This would suggest that an approximation to the desired map of $D$ onto an annulus, obtained by $n$ iterated conformal mappings of simply connected regions, might be constructed by using $n-1$ such mappings to determine the approximation $f_{n-1}(z)$ to $f(z)$, and following this by the map of the interior of $C^{*(n-1)}$ [the image of $C^{*}$ under $Z=f_{n-1}(z)$ ] onto the interior of the unit circle. We shall study the rapidity of convergence of this approximation process.

Let $Z=f_{n}(z)$ be, as in Theorem 1, the approximation to $Z=f(z)$ obtained by $n$ successive conformal maps and reflections. Let $C_{1}^{n}, C^{n}, C^{*_{n}}$ be the images in the $Z$-plane of $C_{1}, C, C^{*}$ respectively under the map $Z=f_{n}(z)$. Let $w=h_{n}(Z)$ map the interior of $C^{*_{n}}$ onto $|w|<1$, with $h_{n}(0)=0$ and $h_{n}^{\prime}(0)>0$, and let $F_{n+1}(z) \equiv h_{n}\left[f_{n}(z)\right]$. Let $w=h(Z)$ be as defined above, and let $F(z) \equiv h[f(z)]$; by Proposition 1, the map $w=F(z)$ takes $D$ onto an annulus.

THEOREM 8. Let the maps $w=F_{n}(z)$ and $w=F(z)$ be as defined above. Then

$$
\left|F(z)-F_{n}(z)\right|<M \rho^{2^{n}}, \quad z \in D,
$$

where $M$ and $\rho<1$ are constants depending only on $D$.

Proof. The functions $\log \left|h_{n}(Z)\right|$ and $\log |h(Z)|$ are Green's functions with pole at $Z=0$ of the domains interior to the contours $C^{*_{n}}$ and $\gamma^{*}$ respectively; we will estimate their difference by means of Hadamard's variational formula. Since $C^{*_{n}}$ and $\boldsymbol{\gamma}^{*}$ have continuously turning tangents, the normal displacement [9] $\delta_{n}(s)$ of $C^{*_{n}}$ with respect to $\gamma^{*}$ is a continuous function of $s$, and Hadamard's formula yields

(13) $\log \left|h_{n}(Z)\right|-\log \left|h^{\prime}(Z)\right|=\frac{1}{2 \pi} \int_{\gamma^{*}} \frac{\partial g(t, Z)}{\partial n_{t}} \frac{\partial g(t, 0)}{\partial n_{t}} \delta_{n}(s) d s_{t}+O\left(\delta_{n}(s)\right)$,

where $t \in \gamma^{*}$, the function $g(t, Z)$ is the Green's function of the interior of $\gamma^{*}$ with pole at $Z$, the normal $n_{t}$ points outward on $\gamma^{*}$, and $Z$ is in the common domain of definition of $h_{n}$ and $h$. By (1) we have, for $z$ exterior to $C_{1}$, 


$$
\begin{aligned}
\left|f_{n}(z)-f(z)\right| & =\left|\left(f_{n}-f_{n+1}\right)+\left(f_{n+1}-f_{n+2}\right)+\cdots\right| \\
& \leqq\left|f_{n}-f_{n-1}\right|+\left|f_{n+1}-f_{n+2}\right|+\cdots \\
& \leqq \frac{K_{1} \cdots K_{n}\left(K_{n+1}+1\right)}{R_{n} R_{n-1}^{2} \cdots R^{2^{n}}}+\frac{K_{1} \cdots K_{n+1}\left(K_{n+2}+1\right)}{R_{n+1} R_{n}^{2} \cdots R^{2^{n+1}}}+\cdots, \\
& \quad \text { with } K_{i}<1, \text { and } R_{n+1}>R_{n}>\cdots>R_{1}>R>1, \\
& \leqq A_{1} / R^{2^{n+1}}, \quad \text { with } A_{1} \text { independent of } n .
\end{aligned}
$$

Set $\rho=1 / R, \epsilon_{n}=\rho^{2^{n}}$, and $\phi_{n}(Z) \equiv \log \left|h_{n}(Z)\right|-\log |h(Z)|$. Let $A$ : represent henceforth constants independent of $n$. Let $\Gamma_{1}$ and $\Gamma_{2}$ be two contours in the $Z$-plane separating $\gamma^{*}$ from $\gamma$ and $\Gamma_{1}$ from $\gamma$ respectively. By applying (14) to $z \in C^{*}$, we obtain

$$
\left|\delta_{n}(s)\right|<A_{2} \epsilon_{n+1},
$$

so that for $n>N_{0}$, the contour $\Gamma_{1}$ lies interior to $C^{* n}$. For $n>N_{0}$ and $Z$ interior to $\Gamma_{1}$, the left-hand side of (13) is defined and since $\Gamma_{1}$ and $\gamma^{*}$ are disjoint, the partial derivatives $\partial g(t, Z) / \partial n_{t}$ and $\partial g(t, 0) / \partial n_{t}$, for $t \in \gamma^{*}$, are uniformly bounded in $Z$. Thus from (13)

$$
\left|\phi_{n}(Z)\right|<A_{3} \epsilon_{n+1}, \quad Z \text { interior to } \Gamma_{1} \text {, all } n>N_{0} .
$$

We next obtain bounds on the conjugate harmonic function $\psi_{n}(Z)$ of $\phi_{n}(Z)$ from the expression

$$
\psi_{n}(Z)-\psi_{n}\left(Z_{0}\right)=\int_{C}\left(-\frac{\partial \phi_{n}}{\partial Y} d X+\frac{\partial \phi_{n}}{\partial X} d Y\right)
$$

where $Z=X+i Y$ and $C$ is an analytic arc interior to $\Gamma_{1}$ joining $Z$ to $Z_{0}$. By definition of $\phi_{n}(Z)$ we have

$$
\psi_{n}(Z) \equiv \arg \left[h_{n}(Z) / h(Z)\right]
$$

whence, by the normalization of $h_{n}$ and of $h$ at $Z=0$, we obtain $\psi_{n}(0)=0$. The interior of $\Gamma_{2}$ is, with its closure, contained in the interior of $\Gamma_{1}$, hence the partial derivatives of the harmonic function $\phi_{n}(Z)$ are uniformly bounded there and, from (15),

$$
\left|\frac{\partial \phi_{n}}{\partial X}\right|,\left|\frac{\partial \phi_{n}}{\partial Y}\right|<A_{4} \epsilon_{n+1}, \quad Z \text { interior to } \Gamma_{2} \text {, all } n>N_{0} .
$$

From (16), therefore

$$
\left|\psi_{n}(Z)\right|<A_{5} \epsilon_{n+1}, \quad Z \text { interior to } \Gamma_{2} \text {, all } n>N_{0} .
$$

We combine the above bounds as follows:

$$
\log \left[h_{n}(Z) / h(Z)\right] \equiv \phi_{n}(Z)+i \psi_{n}(Z)
$$


whence

$$
h(Z)-h_{n}(Z) \equiv h(Z)\left[1-e^{\phi_{n}(Z)+i \psi_{n}(Z)}\right] .
$$

By applying (14) to $z \in C$, we see that, for $n>N_{1}$, the contour $C^{n}$ lies interior to $\Gamma_{2}$. Thus the bounds (15) and (17) are valid for $Z$ on and interior to $C^{n}$ as soon as $n>N_{2}=\max \left(N_{0}, N_{1}\right)$, whence, from (18),

$$
\left|h(Z)-h_{n}(Z)\right|<A_{6} \epsilon_{n+1}, \quad Z \text { on and interior to } C^{n} \text {, all } n>N_{2} .
$$

Now by definition

$$
\begin{aligned}
\left|F(z)-F_{n+1}(z)\right| & =\left|h[f(z)]-h\left[f_{n}(z)\right]+h\left[f_{n}(z)\right]-h_{n}\left[f_{n}(z)\right]\right| \\
& \leqq\left|h[f(z)]-h\left[f_{n}(z)\right]\right|+\left|h\left[f_{n}(z)\right]-h_{n}\left[f_{n}(z)\right]\right| .
\end{aligned}
$$

The function $h(Z)$ is defined and analytic for $Z$ interior to $\gamma^{*}$, thus its derivative is uniformly bounded for $Z$ interior to $\Gamma_{2}$. This fact, combined with (14), yields

$$
\left|h[f(z)]-h\left[f_{n}(z)\right]\right|<A_{7} \epsilon_{n+1}, \quad z \in D,
$$

whence, with (19) we obtain from (20)

$$
\left|F(z)-F_{n+1}(z)\right|<M \epsilon_{n+1}=M \rho^{2^{n+1}},
$$

for $z \in D$ and all $n>N_{2}$. Theorem 8 is established.

\section{BiBLIOGRAPHY}

1. L. Ahlfors and A. Beurling, Invariants conformes et problèmes extrémaux, C. R. Dixième Congrès Math. Scandinaves, 1946, pp. 341-351.

2. L. Ahlfors, Conformal mapping, Lecture Notes at Oklahoma Agricultural and Mechanical College, Summer, 1951.

3. L. Ahlfors and A. Beurling, Conformal invariants, Construction and Applications of Conformal Maps, Proceedings of a Symposium, pp. 243-245; National Bureau of Standards, Appl. Math. Ser. No. 18, Washington, 1952.

4. C. Carathéodory, Conformal representation, Cambridge Tracts in Mathematics and Mathematical Physics, No. 28.

5. D. Gaier, Über ein iterationsverfahren von Komatu zur konformen Abbildung von Ringgebieten, J. Math. Mech. vol. 6 (1957) pp. 865-885.

6. H. Grunsky, Über konforme Abbildungen die gewisse Gebietsfunktionen in elementare Funktionen transformieren. I, Math. Z. vol. 67 (1957) pp. 129-132.

7. J. A. Jenkins, On a canonical conformal mapping of J. L. Walsh, Trans. Amer. Math. Soc. vol. 88 (1958) pp. 207-213.

8. Y. Komatu, Ein alternierendes Approximationsverfahren für konforme Abbildung von einem Ringgebiete auf einen Kreisring, Proc. Japan Acad. vol. 21 (1945) pp. 146-155.

9. Z. Nehari, Conformal mapping, International Series in Pure and Applied Mathematics, New York, MçGraw-Hill, 1952.

10. W. F. Osgood, Lehrbuch der Funktionentheorie. I, Leipzig, 1928.

11. J. L. Walsh, On the conformal mapping of multiply connected regions, Trans. Amer. Math. Soc. vol. 82 (1956) pp. 128-146.

Bell Telephone laboratories, Murray Hill. New Jersey 